\title{
Research on Evolution Process of Credit Risk Contagion Under Degree State Transition
}

\author{
Tianqi Wang ${ }^{1}$, Yi Sun ${ }^{2 *}$
}

\author{
${ }^{1}$ School of Economics and Management, Beijing University of Posts and Telecommunications, Beijing,100876, China \\ ${ }^{2}$ School of Computer Science, Beijing University of Posts and Telecommunications, Beijing,100876, China \\ ${ }^{*}$ Corresponding author. Email: sunyisse@bupt.edu.cn
}

\begin{abstract}
This paper starts from the assumption that the credit relationship network will have a "degree transition", that is, the underlying network is a limited dynamic, and the evolution process of credit risk contagion under the open network system with noise factors is studied. Firstly, under the assumption of dynamic network, the stochastic resonance adiabatic approximation theory is used to derive the probability of moderate change in social networks, and the nonMarkov model of infection probability is constructed. Based on this, the factors such as noise intensity and evolution time are discussed. The impact and discovery of the non-linear characteristics of the credit risk contagion process under the open system, indicating that the evolution process of its contagion is the interaction of the strong interaction between the system movement process and finally through the simulation program to verify the validity of the conclusion. This paper emphasizes the significant adjustment of credit relationship in the occurrence of credit risk events. By controlling the evolution of time, the nonlinear evolution process of credit contagion is more realistic.
\end{abstract}

Keywords: State transition, open network system, credit risk infection

\section{度状态跃迁下的信用风险传染演化过程研究}

\section{王天棋 ${ }^{1}$ 孙艺 $2^{*}$}

\author{
1经济与管理学院, 北京邮电大学 \\ 2 计算机学院 (示范性软件学院), 北京邮电大学 \\ *通信作者邮箱: sunyisse@bupt.edu.cn
}

\section{摘要 :}

本文从信用关系网络会发生 “度状态跃迁” 的假设出发, 即底层网络是有限动态, 研究了融合噪声因素的开 放网络系统下的信用风险传染演化过程。首先在动态网络的假设下, 利用随机共振绝热近似理论推导社会网 络中度的变化概率, 构建了传染概率的非马尔科夫模型, 在此基础上讨论噪声强度和演化时间等因素对演化 过程的影响, 发现信用风险传染过程在开放系统下变化的非线性特点, 说明其传染的演进过程是因素间交互 强作用的系统运动过程最后通过仿真程序, 验证结论的有效性。研究强调了信用关系在信用风险事件发生时 的大幅度调整，通过控制时间演进，更真实地了信用传染的非线性演化过程。

关键词: 度状态跃迁，开放网络系统，信用风险传染

\section{1. 引言}

本文研究来源于对小微企业联保和担保制度所 形成的信贷网络的思考, 小微企业的信用评估由于其 相关数据的缺失难以适应传统信用模型。不同于大中
企业，小微企业间不仅存在复杂的产业关系网络，基 于传统融资所形成的借贷网络也较为复杂。即使通过 担保和联保等增信方式, 仍存在小微企业坏账烂账频 发现象, 特别是由于联保集体中企业的同质性和小微 企业的弱信用体系，极易引发信用风险传染现象。当 
下对于企业的信用研究主要集中在建立小微企业的 信用评价指标体系, 忽视了小微企业的一个信用风险 源头，即风险传染因素带来的信用违约危机，本文试 图构建具有小微企业集合特征的复杂网络模型, 从动 态交互的视角研究小微企业信用。

信用风险传染过程依赖信用个体之间的关联关 系, 时间演化和个体行为, 又基于信用的社会性，信 用风险传染问题较适合以复杂网络相关理论进行研 究。本文提出了基于社会网络结构的信用风险传染演 化模型, 通过随机共振理论加入噪声因素建立开放网 络系统, 量化度跃迁过程, 并利用非马尔科夫过程建 立其系统均衡方程, 在此基础上进行相关因素 (时间、 噪声强度等) 的交互作用分析, 得出相关结论。

\section{2. 相关文献回顾}

相关信用风险传染问题的研究集中在复杂网络 理论基础上, 对网络的节点、边和拓扑结构赋予一定 的实际经济意义,例如基于金融资产之间的实在联系 建立网络，包括业务联系、资产联系和信贷联系等， 同时在经济环境的影响下, 通过信用事件的发生, 个 体之间作为节点产生相应的信用风险传染现象, 复杂 网络可以直观地描述信用风险个体之间的复杂关系, 并可以有效地分析影响风险传递的内生和外生因素。 李永奎（2016）[1] 从资产关联视角并利用复杂网络 和传染病 SIS 理论分析发现, 资产关联关系对关联信 用风险的传染具有双向作用。韩璐（2016）[2]通过 相依性网络分析方法度量上市公司的违约相依度, 证 实了违约传染的资金链路径。钱茜（2018）[3]认为 供应链上信用风险包括信贷信用、商业信用风险和关 联信用风险, 商品的市场价格、供应商生产成本和无 风险利率都会影响供应链上关联信用风险的传染强 度。

信用环境的噪声因素对于信用风险传染过程的 影响也不容忽视。环境噪声为信用网络中的與论信息, 舆论信息往往会引发群体行为, 随机共振理论是指非 线性双稳系统中, 在噪声, 弱信号和系统三者之间的 协同作用下, 实现信号的放大, 在社会学科中主要应 用在與情传播。由于信用违约事件属于原生事件, 而 信用个体之间关系的调整属于衍生事件, 即使是极小 的噪声, 也会引发调整行为的扩大, 两者之间符合共 振模型的基本场景。高杨（2008）[4]发现在嘈杂小 世界网络中, 存在特定的拓扑结构使得系统对于外界 弱信号的放大作用最大。苏继超等（2017）［5]通过 控制噪声强度研究网络與论观点形成, 噪声强度越大, 公众观点收玫值范围越大, 个体行动趋于无序化。信 用风险传染演化过程中, 网络系统内外会发生无效或 低效的信息交换, 基于行为经济学和不完全市场理论, 个体决策会受到信息集合（包括他人决策行为、其他 个体基本信息等）和个体有限理性的影响。

信用主体行动模式决定了信用风险传染模型复 杂网络的属性演化。Chen 和 He [14]构建的信用风险 传染复杂网络模型研究了信用主体的风险态度和抵
御信用风险的能力对节点关系的影响。 Jiang (2018) [15] 从更为微观的角度结合传播动力学 理论和复杂网络分析情绪传染与信用风险传染的耦 合关系, 从而实现了客观信息到主观情绪的转变。现 有模型多从宏观角度分析, 主要通过调整感染比例来 研究风险传染, 少数集中在微观主体的行为和情绪角 度, 而过于微观会给研究带来更多不确定性, 从而影 响结果信度, 本文通过对处于中观层面的复杂网络度 的研究, 而且对于网络中个体信用关系的调整过程描 述是是与否的单刻度, 缺少时间尺度的度量, 现实中 信用关系的调整在信用事件发生时通常在较短时间 即可完成, 从而需要对信用的复杂网络的度属性做更 为细化的假设。

企业等信用个体会在信用事件发生时调整自身 社会关系以规避、分散和转移风险。格兰多利(1995) 等认为企业网络是调节公司之间相互依赖关系的一 种模式。李正彪（2005）[6]企业社会网络是获取社 会资本的基础, 并且在企业的各个阶段都有不同形式 的网络模式, 尤其是中小企业结网动机更强。程恩富

（2002）[7]等针对中国企业的社会网络提出，中国 制度性资源的缺乏是非国有企业利用社会关系因素 作为资源配置的重要原因。所以，在正规有效金融工 具供给不足的环境下, 企业融资更加依赖社会网络。 通过建立双向或单向的借贷关系，当信用事件冲击网 络时, 个体会通过调整社会关系来应对冲击。本文通 过将信用风险传染过程中的信用个体相关行为简化 为个体对社会关系的调整行为, 进一步研究节点度的 分布变化过程, 实现对其演化过程动态模型的建立。

\section{3. 模型假设与构建}

\section{1 模型假设}

本文假设信用风险传染中通过因果关系传染的 部分已经完成, 信用风险传染仅通过个体之间的关联 关系进行, 关联关系越强, 传染速率越大, 初始感染 节点随机确定。市场信用风险持有者的关联关系网络 是一个复杂网络, 其会随着传染过程的发生而变化, 即社会网络中节点与节点之间相连状态会随时间调 整，通过信用信息（包括真信息和舆论信息）和其他 全部信息的交换发生连接和断连, 从而影响社会网络 中节点度的分布。

由于信用个体之间的社会网络具有小世界网络 的特征, 度在特定时刻的分布函数可近似泊松分布, 其分布具有相对稳定性。根据 Burt（1992）[18]对于 企业战略网络稳定性的相关研究，企业声誉，企业间 的信任等社会资本在市场不确定的情况下是维护企 业网络稳定性的主要因素, 当信用事件冲击社会网络 时, 个体对企业间关系网络调整, 可表现为度变化。 本文假设度变化量在正负值两状态分别近似服从高 斯分布, 则两势阱可较好描述企业关系变化趋向的稳 定状态, 并假设度增量为正值时是提高社会关联度, 为负值时为降低社会关联度。绝热近似理论可较好的 
解释离散二态系统的随机共振现象, 本文利用其动力 学模型构建信用风险传染过程的方程式。显然在 $\mathrm{t}$ 时 刻, 度变化量在 0 值两边取值的概率总量之和为 1 。

\section{2 模型建立与推导}

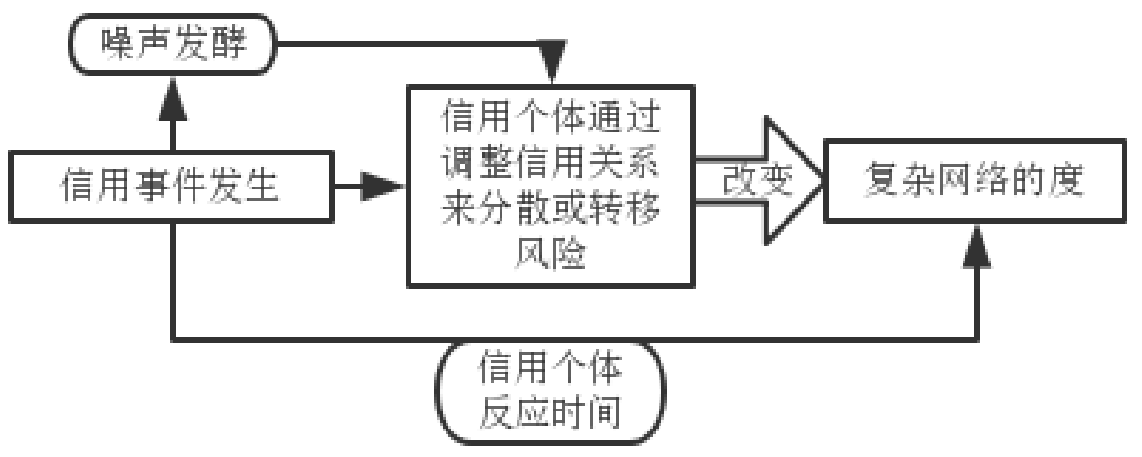

图 1 模型逻辑框架

基于随机共振的绝热近似模型, 计算度增量在噪 声因素影响下, 经过时间 T 之后的变迁概率分布。由 于本文研究的信用状态的跃迁主要关注非系统性因 素下的跃迁, 度跃迁概率模型需要排除大部分的周期 性, 绝热近似模型假设周期信号较弱, 较为适合作为 信用状态跃迁的概率模型。绝热近似模型中, 假设度 增量为 $\mathrm{k}_{\Delta}$, 两个稳定区域可表示为 $(0,+\infty)$ 和 $(0,-$ $\infty)$, 与 $\mathrm{k}_{\Delta}$ 或正或负的取值范围相契合。平均度为 $\overline{\mathrm{k}}$, 其福克一普朗克方程表示如下:

$$
\begin{aligned}
\frac{\partial \mathrm{p}\left(\mathrm{k}_{\Delta}, \mathrm{t}\right)}{\partial \mathrm{t}}=- & \left.\left.\frac{\partial}{\partial \mathrm{k}_{\Delta}} \overline{\mathrm{k}} \mathrm{k}_{\Delta}-\mathrm{k}_{\Delta}{ }^{3}+\mathrm{A} \cos (\Omega \mathrm{t})\right) \mathrm{p}\left(\mathrm{k}_{\Delta}, \mathrm{t}\right)\right] \\
& +\mathrm{D} \frac{\partial^{2}}{\partial \mathrm{k}_{\Delta}{ }^{2}} \mathrm{p}\left(\mathrm{k}_{\Delta}, \mathrm{t}\right)
\end{aligned}
$$

邓定解 $: \mathrm{p}=\mathrm{Ce}^{-\frac{\mathrm{U}\left(\mathrm{k}_{\Delta}\right)}{\mathrm{D}}}, \mathrm{t} \rightarrow+\infty$.

其中 $\mathrm{A}$ 和 $\Omega$ 是周期力参数, $\mathrm{D}$ 为噪声强度, $\mathrm{U}(\mathrm{x})$ 为势函数时, $\mathrm{D}>1$ 时, 噪声输入对于稳定解为数值 缩小效应, $\mathrm{D}<1$, 噪声为扩大效应, 进一步影响两 稳态势阱跃迁概率, 且 $D \neq 0$ 时, 无论周期振幅大小, 度变化量都可实现稳态间跃迁。

显然此模型是双稳态系统, 令势函数为零可以得 到系统的两个势阱点和一个极大值点 $\mathrm{k}_{\Delta}=0$ (非稳定
点）, 两个势阱可以表示度增量的两个基本稳定态即 $\{+,-\}$ 时的期望值, 在社会网络中，信用个体关 联关系随着系统内部的因素影响和系统外部的噪声 影响, 通常在决策中以关联对象的关系优劣为依据, 实现网络的断连和重连, 降低自身与外界的关联以避 免信用传染，或者增强关联关系实现风险的转移和分 散, 从而调整社会网络中信用个体节点的度。

由 Langevin 方程, 可进一步推出运动粒子在两 个势陎之间的跃迁概率。设 $\mathrm{p}_{\mathrm{s}}$ 为度不变概率, 暂且人 文持续重连和持续断连行为都属于状态不变, 即在信 用事件的冲击下依旧保持原行动轨迹; $P\left(\mathrm{k}_{\Delta}>0\right)$ 为 度减概率, $P\left(k_{\Delta}<0\right)$ 为度增概率, 度增和度减的概 率之和为 $\mathrm{p}_{\mathrm{j}}$ 。在信用风险传染模型中, 运动粒子势荫 的变迁可以等价为社会网络中信用个体的行动, 即通 过社会关系变动应对信用风险传染, 包括传染行为和 防御行为, 本文假设传染行为者数和防御行为者数相 加为行动者总数, 则根据随机共振的随机微分方程 (1) 推导易得二维跃迁概率公式 $\mathrm{p}\left(\mathrm{k}_{\Delta}, \mathrm{t}^{\prime} \mid \mathrm{k}_{\Delta}, \mathrm{t}\right)$, 由条件 概率公式 $\mathrm{p}\left(\mathrm{k}_{\Delta}, \mathrm{t}^{\prime} \mid \mathrm{k}_{\Delta}, \mathrm{t}\right) * \mathrm{p}\left(\mathrm{k}_{\Delta}, \mathrm{t}\right)=\mathrm{p}\left(\mathrm{k}_{\Delta}, \mathrm{t}^{\prime}\right)$ 以及度 的近似泊松分布假设, 可以得到度跃迁概率 $\left(\mathrm{k}_{\Delta} \neq 0\right)$ 和度不变 $\left(\mathrm{k}_{\Delta}=0\right)$ 概率, 如下:

$$
\left\{\begin{array}{c}
p_{s}=p(+>+)+\mathrm{p}(->-)=\frac{1}{2}\left(N_{t}^{+}+N_{t}^{-}\right) \sqrt{\frac{2 \pi D}{U^{\prime \prime}\left(k_{+\Delta}\right)}} e^{\left[-\frac{U\left(k_{+}\right)}{D}-R_{0} \tau\right]} \\
p_{j}=p\left(k_{\Delta}>0\right)+\mathrm{p}\left(k_{\Delta}<0\right)=1-p_{s}
\end{array}\right.
$$

其中 $\mathrm{N}_{\mathrm{t}}^{+}+\mathrm{N}_{\mathrm{t}}^{-}$可设定为常数, 即信用个体对信用 风险不敏感的概率, 因为风险敏感度微观行为性较强, 本文不过多的深究微观因素。
信用风险传染模型是一个动态演化过程, 复杂的 网络结构与动力学关联性相互作用, 传统假设节点之 间不存在动力学关联, 度异质性较低, 传染过程为马 
尔科夫过程等，只适用于独立集中网络（供应链）、 社区网络（联保）。本文建立非马尔科夫信用风险传 染模型, 假设信用个体会采取断连和重连信用关系来 防御信用风险的传染, 这也是加入时间间隔 $\tau$ 对传染 率、恢复率和防御措施的采取概率的影响。假设 $\mathrm{M}_{\mathrm{k}}(\mathrm{t})$ 为 $t$ 时刻度为 $k$ 且被传染的个体占网络总量的比例, $\mathrm{P}_{\mathrm{c}}$ 为传染率, $\mathrm{P}_{\mathrm{r}}$ 为恢复率。构建传染过程的非马尔科 夫性公式表示如下:

$=\left(1-\mathrm{M}_{\mathrm{k}}(\mathrm{t})\right) \mathrm{P}_{\mathrm{c}}(\tau)$

$$
\frac{\mathrm{dM}_{\mathrm{k}}\left(\mathrm{t}^{\prime}\right)}{\mathrm{dt}}
$$

$-\mathrm{M}_{\mathrm{k}}(\mathrm{t}) \mathrm{P}_{\mathrm{r}}(\tau$

)

其中 (3) 式右边第一项表示传染个体比例增量, 第二项表示被传染信用风险后恢复正常比例, 为传染 个体比例湮灭量。信用风险传染网络的非马尔科夫性 主要体现在时间到叠加效应, 以 $\tau$ 作为时间强度作用 于于传染率和恢复率。
假设防御个体采取防御行为的驱动仅限于信用 风险信息的交换传播, 不考虑其个体历史经验因素差 异, 个体采取防御行为的概率随信息和时间的积累强 化, 在积累效应强度为 $\gamma$, 信用风险传染过程起始点 防御概率为 $g$ 时, 信用个体采取防御措施的概率为:

$$
=\mathrm{e}^{-{ }^{\tau} \tau} \bigotimes_{0}-\mathrm{e}^{-\gamma \tau}
$$<smiles>C1=[Al][Al]1</smiles>

$+1$

\section{且设恢复率 $P_{r}(\tau)$ 为 $r_{t}{ }_{t}$ 。}

根据信息传播理论，人的行为具有记忆性，随着 传染过程的进行, 个体会根据信息的积累进行决策并 采取行动重连或断连网络, 尤其是从而引起自身 $\mathrm{k}$ 值 的改变, 进而影响 $\mathrm{k}$ 分布, 增加异质性或相反。本文 采用融合时间衰减因素的传播概率模型来表示两个 体之间传染概率如下:

$$
\begin{aligned}
P_{c}(\tau) & =\int_{t_{i}}^{t_{j}} \frac{1}{1+e^{-f\left(i, j_{k}\right)}} \tau *\left(t-t_{i}\right) e^{-\left(\frac{1}{2} \tau *\left(t-t_{i}\right)^{2}\right)} d t \\
& \quad \mathrm{f}\left(\mathrm{i}, \mathrm{j}_{\mathrm{k}}\right)=\ln \left[\frac{1}{\left(\mathrm{p}\left(\mathrm{k}_{\Delta}>0\right)+\mathrm{p}\left(+\mathrm{k}_{\Delta}<0\right)\right)\left(1-\mathrm{\square}_{\mathrm{t}}\right)}-1\right]
\end{aligned}
$$

上述式子表示传染概率的模型较符合传染变化 过程, 即传染率先随时间递增随后衰减, 左边第一项 是根据信用个体度变化的规律, 构建基础传染概率函 数, 并添加其时间效应（非马尔科夫性）。即信用风 险被传染者采取传染行为分散风险时, 根据前文假设, $\mathrm{p}\left(\mathrm{k}_{\Delta}>0\right)+\mathrm{p}\left(\mathrm{k}_{\Delta}<0\right)(1-9)$ 为被传染个体做风险 分散行为的总量占比。
由（2）式等于 0 时取得均衡为: $M_{k}(t)=$ $P_{c}(\tau) /\left(P_{c}(\tau)+P_{r}(\tau)\right)$, 传染率在演进时间的作用 下, 动态的由传染率和恢复率决定, 而下文将以更多 的参数变量来定义它们。将（4）式和（5）式带入, 推导得到关于噪声和时间间隔等相关参数的动力学 方程式。

$$
\begin{aligned}
& M_{k}(\mathrm{t}) \\
& =1-\frac{2 r \mathcal{J}_{t}(\tau)}{\left(N_{t}^{+}+N_{t}^{-}\right) \sqrt{\frac{2 \pi D}{U^{\prime \prime}\left(k_{+\Delta}\right)}} e^{\left[-\frac{U\left(k_{+\Delta}\right)}{D}-\left(R_{0}+\frac{1}{2} t_{i j}(\overline{\mathrm{k}})^{3}\right) \tau\right]}\left(1-\mathcal{J}_{t}\right) \tau * \frac{1}{2} t_{i j}{ }^{2}+2 r \mathcal{J}_{t}(\tau)}
\end{aligned}
$$

\section{3 理论分析}

根据公式 (6), 不考虑 $\mathrm{N}_{\mathrm{t}}^{+}+\mathrm{N}_{\mathrm{t}}^{-}$的时间变动, 且 在公式中作为稳定项作为常值保留, 不作进一步简化。 由 $0<\mathrm{M}_{\mathrm{k}}(\mathrm{t})<1$, 则 $\mathrm{P}_{\mathrm{r}}(\tau)>0$, 说明恢复率一定大 于 0 , 信用个体陷入信用风险危机后采取一定有效措 施可以摆脱风险状态, 且 $P_{c}(\tau)>0$, 信用风险事件发 生后, 一定会向传染过程演进, 即信用风险在非独立 网络中一定会传染, 这与本文假设相一致。

当噪声强度 $D$ 增大时, 分离 $D$ 对 $M_{k}(t)$ 的影响因 子为 $\frac{-1}{\sqrt{\mathrm{D}} \mathrm{e}^{-1 / \mathrm{D}}}$, 且由 $\mathrm{D}>0$ 可知随着噪声强度增加, 对于
确定的度, $M_{k}(t)$ 逐渐降低, 度分布方差增大, 并通 过影响度变化加强其效应, 即信用个体会通过改变关 联关系行为来影响 $\mathrm{M}_{\mathrm{k}}(\mathrm{t})$, 由此说明系统外噪声对于 信用风险的传染会有扩大效应, 这与小世界网络系统 的随机共振理论相关研究 [3] 相契合, 即噪声可作 用在关联关系特定的中强耦合拓扑结构上, 本文构建 的网络特征恰好符合其拓扑结构。

恢复率 $P_{r}(\tau)$ 非线性变化, 在度分布不变时, 其 越大, $M_{k}(t)$ 越小, $g_{t}$ 对传染率也有反作用, 使得传染 率也呈现非线性变化, 进一步印证了网络结构与传染 效应并不是单调线性关系, 金融网络的高连通性可以 降低感染的可能性, 但也会在问题发生时增加感染风 
险。这说明, 社会系统的恢复作用和破坏作用相互作 用，其间的交互演进过程涉及较多的因素，演化速率 也会影响各个时间点的信用网络连接状态。

时间间隔 $\tau$ 可以用于度量度变化过程和传染演 化过程的时间属性，根据式 (1) 可以做出度分布变 化的估计, $\mathrm{k}$ 在度变化量跃迁的过程中, 由 $\sigma(\mathrm{k})$ 的随 机微分方程知 $\sigma(\mathrm{k})$ 在固定时点存在极大值点和稳定 点, 度分布整体先逐步分散 (异质性增加) 后回升并趋 于稳定 $(t \rightarrow \infty)$, 且噪声减少了回归稳定所需时间。 通过式 (5) 对 $\tau$ 求一阶和二阶导, 综合分析后发现 度 $M_{k}(t)$ 并不会如预期随演进过程逐步下降, 演进过 程中, 恢复率会呈现先增后减, $\mathrm{k}_{\Delta}$ 跃迁概率不断下降, 平均度由 $\tau$ 在 $P_{c}(\tau)$ 中的系数 $R_{0}+\frac{1}{2} t_{i j}{ }^{3}$ 可知, 逃逸速 度 $\mathrm{R}_{0}$ 随噪声增大, $\mathrm{t}_{\mathrm{ij}}{ }^{3}$ 随平均度增大而递减, 所以 $\mathrm{M}_{\mathrm{k}}(\mathrm{t})$ 的变化幅度和频率由 $\mathrm{R}_{0}+\frac{1}{2} \mathrm{t}_{\mathrm{ij}}{ }^{3}$ 和噪声强度决 定, 且 $r<1$ 时 (表示社会系统有较差的修复能力) 其 效应越强。

综上所述, 信用事件发生之后信用个体的反应时 间通过时间积累效应作用在恢复率和传染率上, 并通 过交叉效应影响信用事件的进一步发现; 信用环境中 與论噪声信息的增加信用事件的发酵也会因不同的 度跃迁模式而不同。

\section{4. 仿真实验}

\section{1 度跃汗对信用风险传染过程的影响}

利用 netlogo 软件设计仿真实验, 参数设定如 下: 根据 2018 年债券市场主体信用评级迁移矩阵, 定义信用评级下调 3 个等级代表信用事件发生, 2018 年整年发生信用状态大幅下调的企业数量达到了 35 家, 频率接近 1\%，一般联保企业团体以 5-6 家企业 为主, 在仿真程序设定中, 根据真实数据假设初始信 用违约者比例为 $1 \%$, 度的初始值为 5 。根据信用个体 反应速度和免疫率值将复杂网络分为成熟, 半成熟和 成熟系统, 越成熟的信用个体越能较早地觉察信用违 约事件的发生从而采取行动。当信用个体采取行动的 比例达到一定程度时, 设定为群体行动时点, 从而可 以将公式 (4) 中行动比率的动态值化为较为简明的 静态值。不考虑噪声强度的变化, 且调整过程都为先 断连再重连, 检验度跃迁对信用风险传染过程的影响 程度:

表 1 仿真参数设置

\begin{tabular}{|c|c|c|c|c|c|c|c|}
\hline \multicolumn{8}{|c|}{ 仿真参数设定 } \\
\hline & $\begin{array}{c}\text { 个体调整信用䏌 } \\
\text { 系时点 }\end{array}$ & 节点数 & 度 & $\begin{array}{c}\text { 初始违约 } \\
\text { 节点数 }\end{array}$ & $\begin{array}{c}\text { 违约传染 } \\
\text { 概率\% }\end{array}$ & 恢复率\% & 免疫率\% \\
\hline 度不变模型 & 无 & 300 & 5 & 3 & 3.5 & 4 & 20 \\
\hline \multirow{2}{*}{$\begin{array}{c}\text { 不成熟信用 } \\
\text { 系统 }\end{array}$} & 200 & 300 & 3 & 3 & 2 & 5 & 30 \\
\hline & 500 & 300 & 6 & 3 & 4 & 5 & 35 \\
\hline \multirow{2}{*}{$\begin{array}{c}\text { 半成熟信用 } \\
\text { 系统 }\end{array}$} & 100 & 300 & 3 & 3 & 2 & 5 & 30 \\
\hline & 500 & 300 & 6 & 3 & 4 & 5 & 35 \\
\hline \multirow{2}{*}{$\begin{array}{c}\text { 成熟信用系 } \\
\text { 统 }\end{array}$} & 50 & 300 & 2 & 3 & 3 & 5 & 35 \\
\hline & 300 & 300 & 4 & 3 & 3 & 5 & 40 \\
\hline
\end{tabular}




\section{Network Status}
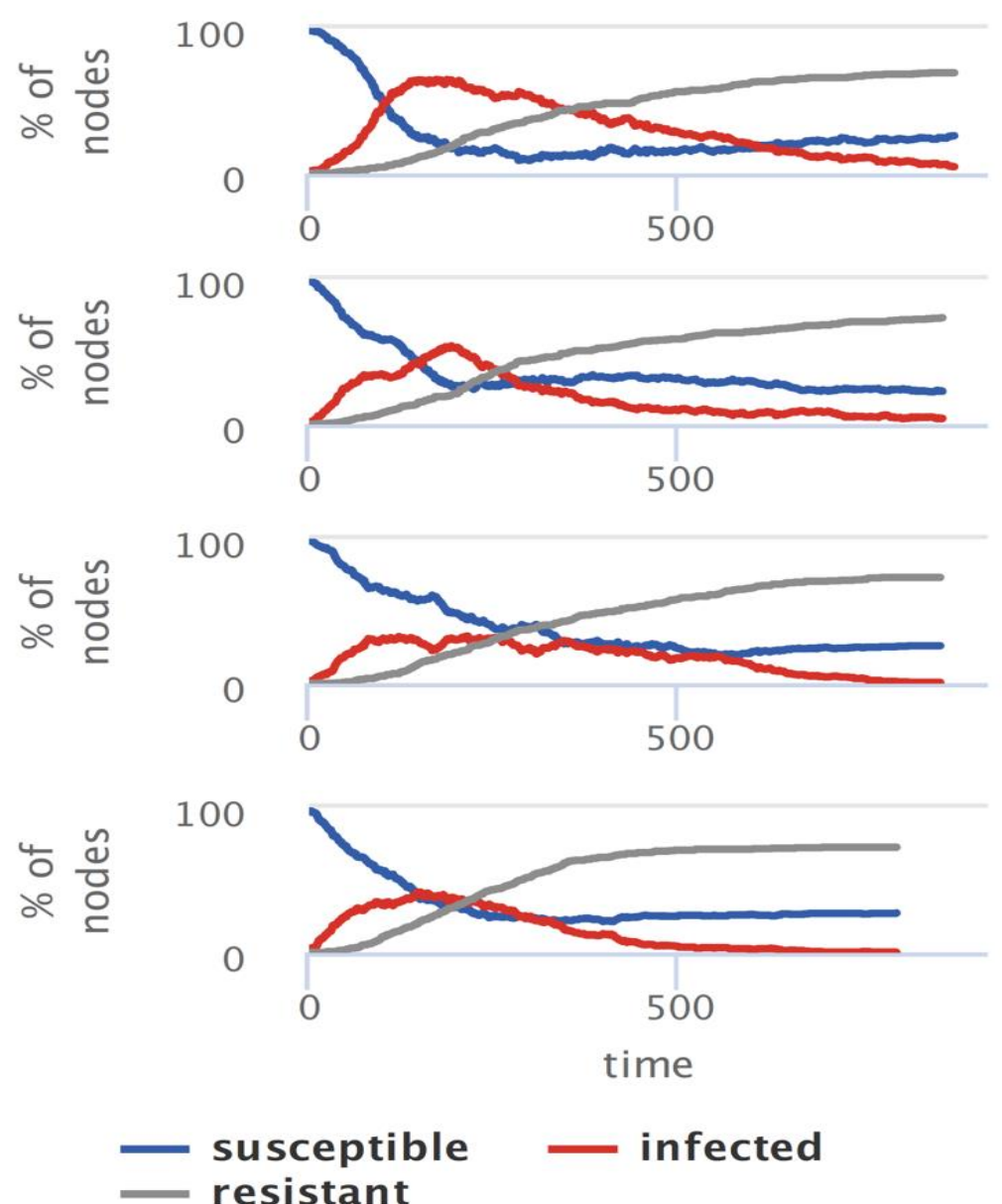

图 2 度跃迁仿真结果

仿真结果如下: 通过不成熟和半成熟信用系统的 比较发现，对信用事件反应敏锐可有效避开群体违约 的峰值, 将信用传染程度限制在可控范围之内; 成熟 信用系统通过假设其在信用事件发酵初期时, 信用个 体集体断连信用关系以避免信用风险传染, 在信用事 件平息前中期即开始重连行为, 恢复正常信用秩序, 不仅可降低信用违约群体事件的最大势能, 也可以在 最短的时间内有效的实现信用事件的平息。更值得注 意的是, 虽然设定的免疫率参数差别较大, 但是平稳 状态下的免疫群体比例在各程度信用体系中表现相 近。

\section{2 噪声在信用复杂网络中的作用}

构建基本信噪比公式检验噪声强度对上节仿真 结果做进一步的检验, 由于信用系统同质性较高, 将 高耦合复杂网络作为底层网络, 通过图表发现, 噪声 在高耦合网络中进一步扩大了耦合效应, 三个系统 表现出同步行为, 但是信噪比的峰值点所对应的噪 声强度不同, 越成熟的信用系统对于微小的噪声反 应越灵敏, 而峰值也越小。 


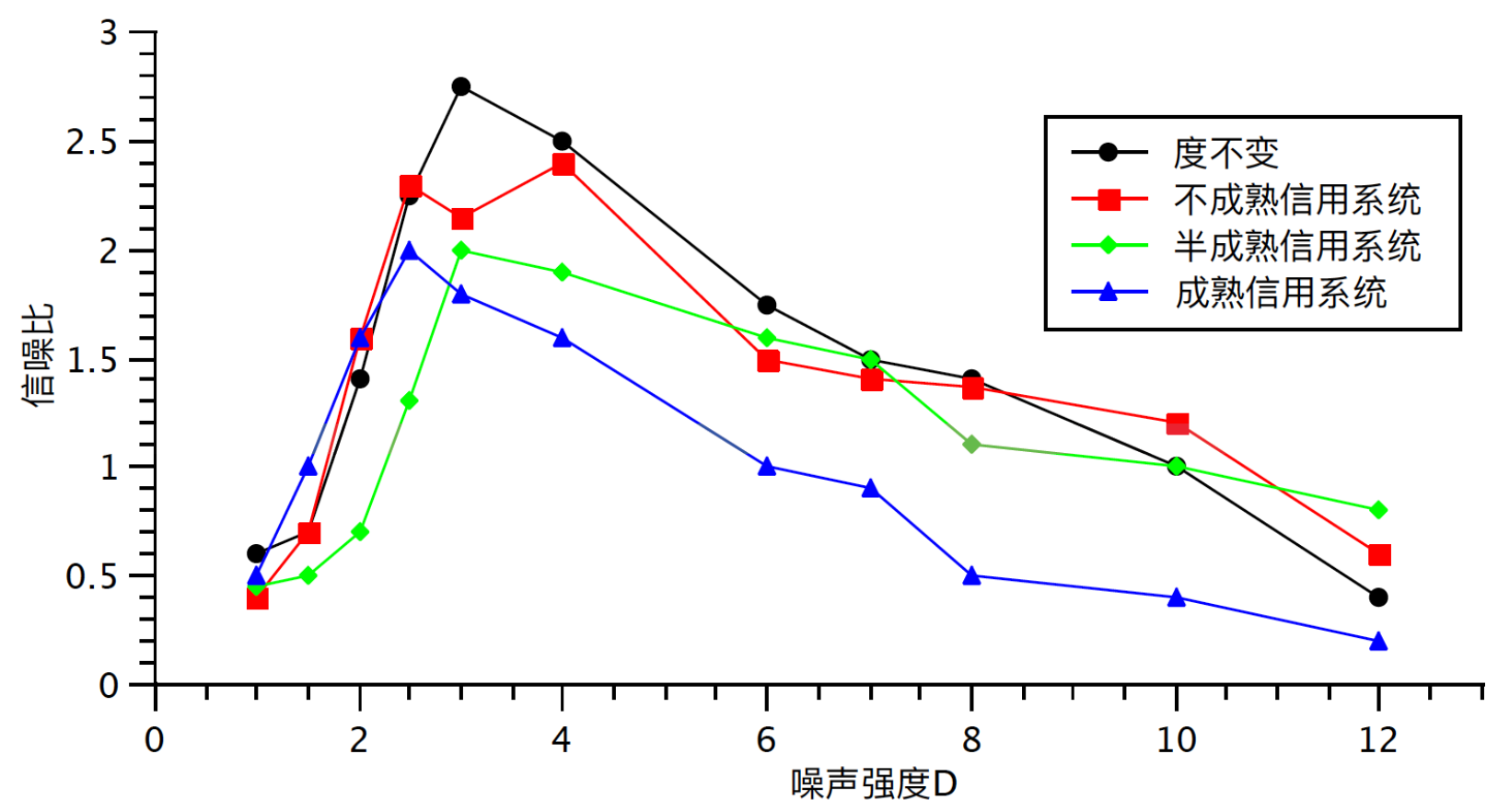

图 3 度跃迁仿真结果

\section{5. 结论}

信用传染过程是一个系统演化问题, 在研究个体 信用风险时, 不应忽略噪声的影响和企业间关联度的 变化。本文利用随机共振理论实现加入信用环境噪声, 并用绝热近似模型模拟资产配置过程基于信用状态 不变的假定, 本文通过建立信用风险传染的非马尔科 夫过程, 并加入系统外噪声, 构建了一个开放式信用 风险传染演化系统, 并关注于重点精神的下的信用风 险传染的演化过程, 很难确定其信用传染过程的时间 拐点和极值点, 但系统中的交互作用都可进行局部解 释, 并且稳定的系统参数会使得社会系统再次恢复均 衡状态。

\section{6. 建议}

金融机构在对小微企业授信的过程中, 应当进行 系统性的评价, 包括对信用环境中各种信息质和量的 评估, 以及对企业社会关联度变化的监测。信用风险 传染现象在小微企业网络中极易发生, 小微企业管理 者能力和信用水平参差不齐, 对信息的辨识度和对风 险的应对能力较弱, 传统信用评估模型仅适用于对环 境敏感度低, 财务稳定性强并且依靠财务状况有应对 风险可能性的企业。小微企业经营绩效依赖于其所处 的社会网络状况和经济信用环境, 其外部性较强, 金 融机构在进行小微企业信用评估时, 应降低财务数据 比重, 结合信用风险传染演化模型进行时间上的管理, 实现动态评估。积极推出适合小微企业的金融产品, 并设计合理的产品内部机制, 降低小微企业联保等的 借贷风险。

小微企业在管理信用风险上应注意降低自身财 务状况对外界的依赖度, 通过合理常规渠道融资, 提
高对风险管理产品的使用意识, 利用恰当的风险分散 手段, 避免因非经营因素导致的破产或财务困境。小 微企业管理者应时刻关注国家政策, 树立正确的价值 观和经营目标, 减少无效信息的错误引导带来的损失, 避免群体行为的发生。

政府在制定小微企业扶持政策时, 应加强对小微 企业借贷环境的管理。净化小微企业借贷环境, 鼓励 正规金融机构设计并推出针对小微企业的金融产品。 寻求民间借贷机构存在的合理形式, 并将其置于金融 监管之下。政府相关部门应注意民间信用信息舆论管 理，增强政府政策可预测性，及时遏制不实消息的传 播, 避免集体恐慌事件。

\section{项目基金}

本文为河北省重点研发项目 (No. 20313701D), 教育部产学合作合作教育项目（No. 201902218004） ( No.201902024006 ) ( No.201901197007 ) （No. 201901199005），教育部产学研合作教育项目 (No. 201901197001), 北京邮电大学教改项目 (No. 500520096 ）的成果之一。

\section{REFERENCES}

[1] Li Yongkui. Research on the Contagion Delay Effect of Associated Credit Risk Based on Complex Networks [D]. University of Electronic Science and Technology of China, 2016.

[2] Han Lu. Research on Topological Structure-based Contagion by Default [A]. Uncertain System Branch of China Operations Research Society. 
Proceedings of the 14th China Uncertain System Annual Conference and the 18th China Youth Information and Management Scholars Conference [C]. Uncertain System Branch of Chinese Society for Operations Research: Department of Mathematical Sciences, Tsinghua University, 2016: 9.

[3] Qian Qian. Research on the Contagion Effect and Management and Control Strategies of Linked Credit Risk [D]. University of Electronic Science and Technology of China, 2018.

[4] Gao Yang, Li Qianshu. Stochastic resonance in noisy small-world networks [J]. Journal of Molecular Science, 2008(04):246-249.

[5] Su Jichao, Zhu Hengmin. Research on the guidance of network public opinion under noise interference[J]. Journal of Information, 2017, 36(10): 91-96+122.

[6] Cheng Enfu, Peng Wenbing. Social network: a new form of enterprise resource allocation [J]. Journal of Shanghai Administration Institute, 2002(02): 79-90.

[7] Zhou Donghao, Han Wenbao, Wang Yongjun. Social network information dissemination model based on nodes and information characteristics [J]. Computer Research and Development, 2015, 52(01): 156-166.

[8] Ming Tingfeng, Zhang Yongxiang, Li Jing. Selfoptimization method and application of stochastic resonance model structural parameters[J]. Journal of Tianjin University (Natural Science and Engineering Technology Edition), 2014, 47(10): 886-891.

[9] Chen Tingqiang, He Jianmin. Research on Credit Risk Contagion Model Based on Complex Networks[J]. China Management Science, 2014, 22(11):1-10.

[10] Lan Guolie. The statistical properties of disease transmission in complex networks with given degree distribution[J]. Journal of Guangzhou University (Natural Science Edition), 2018, 17(03): $1-5$.

[11] Liu Jiubiao. The average domain model of default contagion based on Markov method[J]. Economic Mathematics, 2017, 34(02): 89-94.

[12] Li Yenan. Research on public opinion resonance phenomenon based on stochastic resonance model [D]. China University of Geosciences (Beijing), 2018.

[13] Li Zhengbiao. Research on the social network of corporate growth [D]. Sichuan University, 2005.[14] P. Gai and S. Kapadia, "Contagion in financial networks," Journal of Economic
Literature, vol. 466 , no. 2120 , pp. $2401-2423$, 2010.

[15] Shanshan Jiang, Hong Fan,Credit risk contagion coupling with sentiment contagion,Physica A: Statistical Mechanics and its Applications, Volume 512,2018,Pages 186-202,ISSN 0378-4371.

[16] S. Battiston, J. D. Farmer, A. Flache et al., "Complexity theory and financial regulation," Science, vol. 351, no. 6275, pp. 818- 819, 2016.

[17] D. Acemoglu, A. E. Ozdaglar, and A. Tahbazsalehi, Systemic Risk and Stability in Financial Networks. Nber Working Papers, no. 18727, 2013Department of Economics, Massachusetts Institute of Technology,no.18727,2013, Cambridge, MA, 2013.

[18] Burt,Ronald. Structure Holes: the social of competition. MA: Harvard University Press.1992:62-80.

[19] T.-Q. Chen and J.-M. He, "A network model of credit risk contagion," Discrete Dynamics in Nature and Society, vol. 2012, Article ID 513982, 13 pages, 2012.

[20] A.-C. Hüser, "Too interconnected to fail: a survey of the interbank networks literature," The Journal of Network Theory in Finance, vol. 1, no. 3, pp. 1-50, 2015. 